\title{
A Model Based Poisson Point Process for Downlink Cellular Networks Using Joint Scheduling
}

\section{Sinh Cong Lam \& Kumbesan Sandrasegaran}

Wireless Personal Communications

An International Journal

ISSN 0929-6212

Wireless Pers Commun

DOI 10.1007/s11277-019-06353-7
Volume 106 Number 1 May (I) 2019 FIRST

Wireless Personal Communications

An International Journal

Special Issue: Social Innovation for Sustainable Infrastructure (SISI)

Guest Editors: Ramjee Prasad · Marina Ruggieri

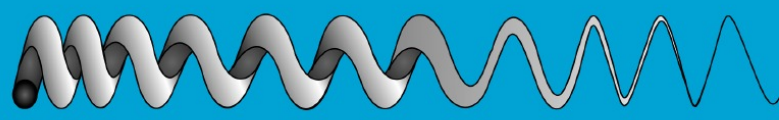

黛 Springer 
Your article is protected by copyright and all rights are held exclusively by Springer Science+Business Media, LLC, part of Springer Nature. This e-offprint is for personal use only and shall not be self-archived in electronic repositories. If you wish to selfarchive your article, please use the accepted manuscript version for posting on your own website. You may further deposit the accepted manuscript version in any repository, provided it is only made publicly available 12 months after official publication or later and provided acknowledgement is given to the original source of publication and a link is inserted to the published article on Springer's website. The link must be accompanied by the following text: "The final publication is available at link.springer.com". 


\title{
A Model Based Poisson Point Process for Downlink Cellular Networks Using Joint Scheduling
}

\author{
Sinh Cong $\operatorname{Lam}^{1} \mathbb{D} \cdot$ Kumbesan Sandrasegaran ${ }^{2}$
}

(c) Springer Science+Business Media, LLC, part of Springer Nature 2019

\begin{abstract}
This paper proposes a model based on a random cellular network to analyse performance of Joint Scheduling in which a typical user measures signal-to-interference-plus-noise ratio (SINR) on different resource blocks from $K$ nearest BSs in order to find out the BS with the highest SINR to establish communication. The paper derives the general form of average coverage probability of a typical user in the case of $K>2$ and its close-form expression in the case of $K=2$. The analytical results which are verified by Monte Carlo simulation indicates that (1) using the Joint Scheduling can improve the user's performance up to $34.88 \%$ in the case of the path loss exponent $\alpha=3$; (2) the effect of the density of BSs on the user association probability is infinitesimal.
\end{abstract}

Keywords Poisson point process $\cdot$ Joint scheduling $\cdot$ Coverage probability

\section{Introduction}

In a LongTerm Evolution-Advanced (LTE-A) cellular network, coordinated multipoint (CoMP) transmission and reception such as Joint Scheduling [1] is a promising technique that can enhance the quality of the received signals as well as mitigate the effects of interference.

In a Joint Scheduling network with a cluster size of $K$, every $K$ adjacent BSs are grouped into a cluster in which the Channel State Information (CSI) is exchanged within this cluster. Conventionally, a typical user measures SINR from $K$ nearest BSs and selects the BSs with the highest SINR as the serving BS. Hence, the downlink SINR of the typical user is given by $\max \left(\operatorname{SINR}_{1}, \ldots, \operatorname{SINR}_{K}\right)$. Fig. 1 is an example of Joint Scheduling with the number of coorinated BSs $K=3$.

In the literature, there was an association approach that allows the user connect to the strongest BS which is called flexible user association [2, 3]. In these works, the user measures the downlink SINR from all BSs and select the BS with highest average

Sinh Cong Lam

lamsinhcong@gmail.com

Kumbesan Sandrasegaran

Kumbesan.Sandrasegaran@uts.edu.au

1 VietNam National University - Hanoi, University of Engineering and Technology, Hanoi, Vietnam

2 University of Technology Sydney, Sydney, Australia 
received-signal strength as the serving BSs. The main differences between Joint Scheduling and flexible association approach are as follows: (1) the flexible association approach only considers the transmit power of BSs and the path loss. Thus, the user only connects to the nearest BS when all BSs transmit at the same power. (2) the Joint Scheduling takes the instantaneous transmission conditions such as fading, intercell interference in the association solution.

In the literature, a lot of research works have been conducted to evaluate the performance of Joint Scheduling in a hexagonal network layout only, which were surveyed in [4]. To the best of our knowledge, work on performance analysis of Joint Scheduling in Poisson point process (PPP) network layout [5] was only conducted in [6]. In this paper, the worst case user with equal distances to three nearest BSs was studied. In addition, the paper assumed that the worst case user measures SINR on the same resource block (RB), which causes all measured signals at the typical user to have the same interference. Thus, the serving BS was selected according to fading channels between the typical user and BSs only. This assumption may be infeasible in practical networks since it may be impossible to find a RB which is free in two adjacent cells at the same time.

In this paper, the performance of the typical user who is located randomly in the cellular network using Joint Scheduling is investigated. Instead of assuming that the user measures SINR on the same RB, the typical user in this work observes $K$ values of SINRs on $K$ different RBs from $K$ BSs. Take $K=2$ for example, SINR from the BS 1 on RB $m$ and BS 2 on $\mathrm{RB} n(m \neq n)$ are observed. Since fading channels vary between RBs, the interference power of the measured SINR from these BSs are different though all signals are affected by interference originating from the same BSs. Thus, the selection of a serving BS depends on various network parameters such as the fading channels, transmit power of BSs, and distance from the typical user to BSs.

\section{Network Model}

\subsection{Network Topology}

We consider the cellular network using Joint Scheduling with a cluster size of $K$ in which the BSs are distributed according to a PPP with density $\lambda$.

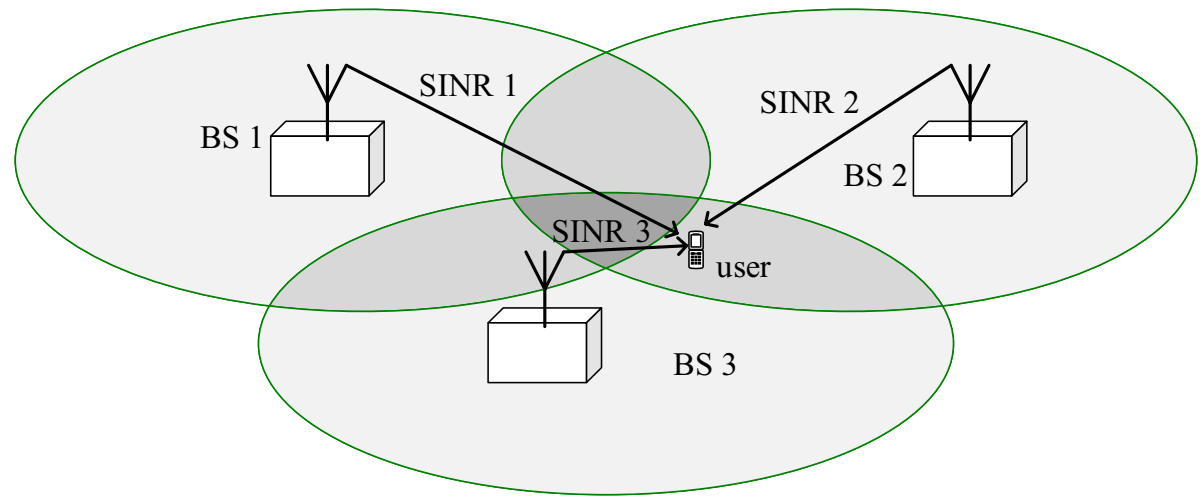

Fig. 1 An example of joint scheduling with $K=3$ 
The typical user is assumed to be located at the origin. We denote $r_{k}$ as the distance from the typical user to the BS $k$ which is a random variable whose the probability density function (PDF) is given by

$$
f_{R_{k}}\left(r_{k}\right)=\frac{2(\pi \lambda)^{k}}{(k-1) !} r_{k}^{2 k-1} e^{-\pi \lambda r_{k}^{2}}
$$

The joint PDF of $R_{1}, R_{2}, \ldots$, and $R_{K}$ in which $R_{1}<R_{2}<\cdots<R_{K}$ is defined by $f_{R_{1}, \ldots, R_{K}}\left(r_{1}, \ldots, r_{K}\right)$ which is given by [7]

$$
f_{R_{1}, \ldots, R_{K}}\left(r_{1}, \ldots, r_{K}\right)=(2 \pi \lambda)^{K} e^{-\pi \lambda r_{K}^{2}} \prod_{m=1}^{K} r_{m}
$$

Therefore, the joint PDF of any $N$ random variables in $S$ which is a subset of $\{1,2, \ldots, K\}$ is denoted by $f_{S}(S)$ and obtained by integrating $K-N$ integrals of $f_{R_{1}, \ldots, R_{K}}\left(r_{1}, \ldots, r_{K}\right)$ with respect to $r_{j}\left(r_{j} \in S^{c}\right)$ by the following equation

$$
f_{S}(S)=(2 \pi \lambda)^{K} \int \cdots \int e^{-\pi \lambda r_{K}^{2}} \prod_{m=1}^{K} r_{m} \prod_{j \in S^{c}} d r_{j}
$$

in which $S^{c}$ is the complementary set of $S, S^{c}=\{1,2, \ldots, K\} \backslash\{S\}$, the bounds of integrations satisfy the following rule: $0<r_{1}<\cdots<r_{K}<\infty$.

\subsection{The Downlink Model}

In a LTE-Advanced network using Joint Scheduling, the typical user experiences interference from all active BSs which can be separated into: (1) Intra-Cluster BSs which includes the BSs within the associated cluster, and (2) Inter-Cluster BSs which includes BSs belonging to other clusters. Hence, the downlink interference at the typical user associated with BS $k$ can be stated as

$$
I_{k}=\underbrace{\sum_{j=1, j \neq k}^{K} P_{j} g_{j}^{(k)} r_{j}^{-\alpha}}_{\text {Intra-Cluster Interference }}+\underbrace{\sum_{j \in \theta^{c}} P_{j} g_{j}^{(k)} r_{j}^{-\alpha}}_{\text {Inter-Cluster Interference }}
$$

in which $g_{j}^{(k)}$ and $r_{j}$ are the power channel gain and distance from the typical user to interfering BS $j$ whose transmit power is $P_{j} ; \alpha$ is the path-loss exponent; $\theta^{c}$ is the set of interfering BSs which belong to adjacent clusters. We denote $\theta$ is the set of BSs in the network, then $\theta=\theta^{c} \bigcup\{1,2, \ldots, K\}$.

Since the BSs in a given cluster fully exchange the channel state information, the IntraCell Interference can be controlled by the scheduling mechanism. Meanwhile the InterCluster Interference may not be controlled. For simplicity, we assume that the typical user only experiences Inter-Cluster Interference. Furthermore, the transmit power of the BS in the cellular network is usually much greater than Gaussian noise, then Gaussian noise can be neglected. Thus, the downlink signal-interference-ratio (SIR) from BS $k$ is given by

$$
\operatorname{SIR}_{k}=\frac{P_{k} g_{k}^{(k)} r_{k}^{-\alpha}}{\sum_{j \in \theta^{c}} P_{j} g_{j}^{(k)} r_{j}^{-\alpha}}
$$


We assume that all BSs transmit at the same power, thus the serving signal of the typical user is given by

$$
S I R=\max _{1 \leq k \leq K}\left(\frac{g_{k}^{(k)} r_{k}^{-\alpha}}{\sum_{j \in \theta^{c}} g_{j}^{(k)} r_{j}^{-\alpha}}\right)
$$

In this paper, each fading channel has a unit power and follows a Rayleigh fading distribution, thus the channel power gain $g_{j}^{(k)}$ is an exponential distribution with PDF $\gamma(g)=\exp (-g)$.

\section{Average Coverage Probability}

The average coverage probability of the typical user for a given coverage threshold $T$ is defined as the probability in which the received SIR is greater than the coverage threshold $T$.

$$
P(T)=\mathbb{P}(S I R>T)
$$

The average coverage probability can be evaluated by the following steps

$$
\begin{aligned}
P(T) & =\mathbb{P}\left(\max _{1 \leq k \leq K}\left(\frac{g_{k}^{(k)} r_{k}^{-\alpha}}{\sum_{j \in \theta^{c}} g_{j}^{(k)} r_{j}^{-\alpha}}\right)>T\right) \\
& =1-\mathbb{P}\left(\max _{1 \leq k \leq K}\left(\frac{g_{k}^{(k)} r_{k}^{-\alpha}}{\sum_{j \in \theta^{c}} g_{j}^{(k)} r_{j}^{-\alpha}}\right)<T\right] \\
& =1-\mathbb{E}\left[\prod_{1 \leq k \leq K} P\left(\frac{g_{k}^{(k)} r_{k}^{-\alpha}}{\sum_{j \in \theta^{c}} g_{j}^{(k)} r_{j}^{-\alpha}}<T\right)\right]
\end{aligned}
$$

With the assumption that all fading channels are independent Rayleigh random variables, the average coverage probability is given by

$$
\begin{aligned}
P(T) & =1-\mathbb{E}\left[\prod_{1 \leq k \leq K}\left(1-\prod_{j \in \theta^{c}} e^{-T r_{k}^{\alpha} r_{j}^{-\alpha} g_{j}^{(k)}}\right)\right] \\
& =\mathbb{E}\left[\sum_{S}(-1)^{N+1} \prod_{k \in S} \prod_{j \in \theta^{c}} e^{-T r_{k}^{\alpha} r_{j}^{-\alpha} g_{j}^{(k)}}\right]
\end{aligned}
$$


in which $S$ is the subset of $\{1,2, \ldots, K\}$ and $S \neq \emptyset, N$ is the number of elements of $S$. Thus, the average coverage probability can be re-written as the following equations

$$
\begin{aligned}
P(T) & =\mathbb{E}\left[\sum_{S}(-1)^{N+1} \prod_{j \in \theta^{c}} \prod_{k \in S} \mathbb{E}_{G_{j}^{(k)}}\left[e^{-T r_{k}^{\alpha} r_{j}^{-\alpha} g_{j}^{(k)}}\right]\right] \\
& =\mathbb{E}\left[\sum_{S}(-1)^{N+1} \mathbb{E}_{\theta^{c}}\left[\prod_{j \in \theta^{c}} \prod_{k \in S} \frac{1}{1+\operatorname{Tr}_{k}^{\alpha} r_{j}^{-\alpha}}\right]\right]
\end{aligned}
$$

By employing the properties of probability generating function (PGF) and reminding that the distance from any interfering BS to the typical user must be greater than $r_{K}$, we obtain

$$
\begin{aligned}
& P(T)=\mathbb{E}\left[\sum_{S}(-1)^{N+1} e^{-2 \pi \lambda \int_{r_{K}}^{\infty} t\left[1-\prod_{k \in S} \frac{1}{1+T_{k}^{\alpha} t^{\alpha-\alpha}}\right] d t}\right] \\
& =(2 \pi \lambda)^{K} \sum_{S}(-1)^{N+1} \int_{0}^{\infty} \int_{r_{1}}^{\infty} \cdots \int_{r_{K-1}}^{\infty} e^{-\pi \lambda r_{K}^{2}-2 \pi \lambda \int_{r_{K}}^{\infty} t\left[1-\prod_{k \in S} \frac{1}{1+T_{k}^{\alpha} t^{-\alpha}}\right] d t} \\
& \times \prod_{j=1}^{K} r_{j} d r_{K-j+1}
\end{aligned}
$$

in which Eq. (8) is the result of taking the expected values of $N$ random variables, $\left(R_{1}, R_{2}, \ldots, R_{K}\right)$, whose joint PDF is defined in Eq. (3).

By employing changes of variable $t^{2}=r_{K} y$ and $x_{j}=\pi \lambda r_{j}^{2},(1 \leq j \leq K)$, we obtain

$$
P(T)=\sum_{S}(-1)^{N+1} \int_{0}^{\infty} \int_{x_{1}}^{\infty} \cdots \int_{x_{K-1}}^{\infty} e^{-x_{K}-x_{K} \int_{1}^{\infty}\left[1-\prod_{k \in S} \frac{1}{1+T_{x}^{\alpha / 2}\left(x_{K} y\right)^{-\alpha / 2}}\right] d y} \prod_{j=1}^{K} d x_{K-j+1}
$$

Equation (9) provides the most important result of this paper which derives the average coverage probability of the typical user. It is interesting that the average coverage probability does not depend on the density of BS, which is consistent with the conclusion for the cellular network without Joint Scheduling [8].

A special case of Joint Scheduling with $K=2, S \subset\{1,2\}$ and $S \neq \emptyset$ By employing a change of variable $t=\frac{r_{1}}{r_{2}}$, the average coverage probability is obtained by the following equation

$$
P(T)=\int_{0}^{\infty} r_{2} e^{-r_{2}}\left[\int_{0}^{1} e^{-r_{2} v(T, t)} d t+e^{-r_{2} v(T, 1)}-\int_{0}^{1} e^{-r_{2} \frac{t^{\alpha / 2} \nu(T, t)-\nu(T, 1)}{t^{\alpha / 2}-1}} d t\right] d r_{2}
$$

in which $v(T, t)=\int_{1}^{\infty} \frac{T t^{\alpha / 2} y^{-\alpha / 2}}{1+T t^{\alpha / 2} y^{-\alpha / 2}} d y$.

In Eq. 10, the infinite integral has a suitable form of Gauss-Legendre Quadrature while the integral defined from $[0,1]$ can be approximated by using Gauss-Laguerre Quadrature. Hence, the average coverage probability can be approximated by 


$$
\begin{aligned}
P(T) \approx & \sum_{j=1}^{N_{G L}} w_{j} t_{i}\left[\sum_{i=1}^{N_{G}} \frac{c_{i}}{2} e^{-t_{j} v\left(T, \eta_{i}\right)}+e^{-t_{j} v(T, 1)}\right] \\
& -\sum_{j=1}^{N_{G L}} w_{j} t_{j} \sum_{i=1}^{N_{G}} \frac{c_{i}}{2} e^{-\zeta_{j}\left[\frac{\eta_{i}^{\alpha / 2} v\left(T, \eta_{i}\right)-v(T, 1)}{\eta_{i}^{\alpha / 2}-1}\right]}
\end{aligned}
$$

where $N_{G L}$ and $N_{G}$ are the degrees of the Laguerre and Legendre polynomial, $t_{i}$ and $w_{i}, c_{i}$ and $x_{i}$ are the $i$-th node and weight, abscissas and weight of the corresponding quadratures; $\eta_{j}=\frac{x_{i}+1}{2}$.

Furthermore, the integral $v(T, t)$ can be presented as the difference of two integrals $I_{0}$ and $I_{1}$ which are defined on intervals $[0, \infty]$ and $[0,1]$, respectively. While the first one, $I_{0}$, is evaluated by employing changes of variables $\gamma=T t^{\alpha / 2} y^{-\alpha / 2}$ and using the properties of Gamma function, the second one $I_{1}$ is approximated by Gauss-Legendre rule. Hence, $v(T, t)$ can be approximated by [9]

$$
v(T, t) \approx \frac{2 t T^{2 / \alpha}}{\alpha} \frac{\pi}{\sin \left(\frac{2 \pi}{\alpha}\right)}-\sum_{i=1}^{N_{G}} \frac{c_{i}}{2} \frac{T t^{\alpha / 2}}{\left(\frac{x_{i}+1}{2}\right)^{\alpha / 2}+T t^{\alpha / 2}}
$$

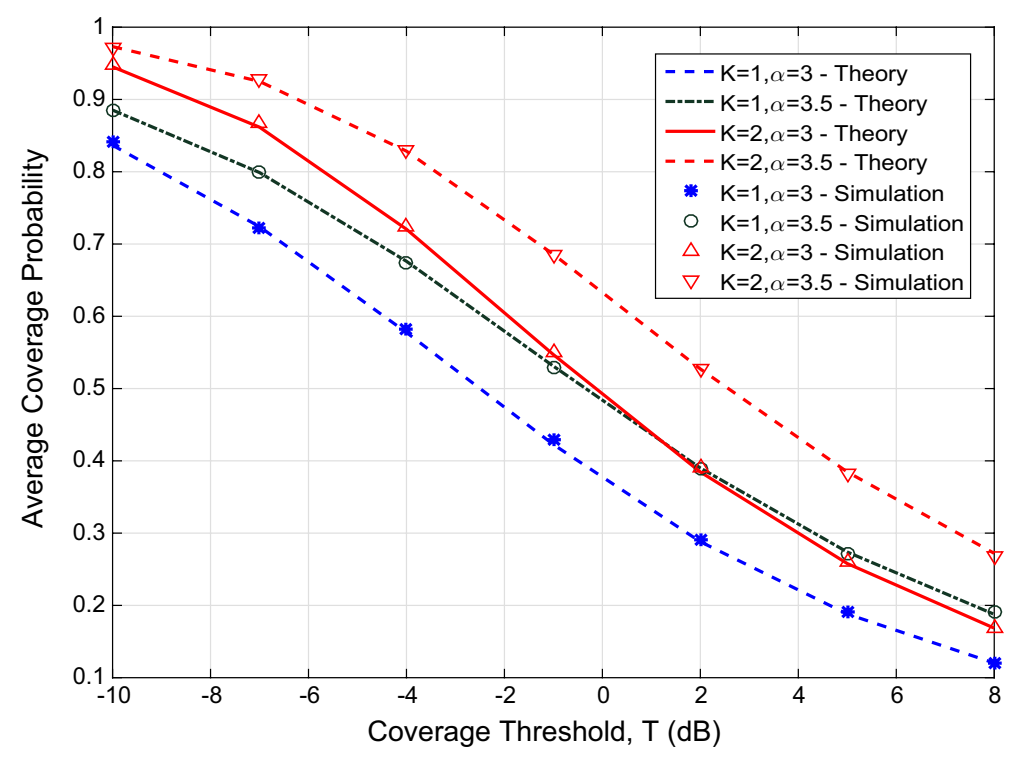

Fig. 2 A comparison between analytical and Monte Carlo simulation results 


\section{Simulation}

\subsection{Validation of the Analytical Results}

In this section, the Monte Carlo simulation results are used to verify the analytical results in Eq. (9) in the cases of $\alpha=3,3.5$ and $K=1,2$. The simulation scenario is set up as follows

- The network cover a globular area with a radius of $\mathrm{R}$ in which $R \rightarrow \infty$

- The density of BSs is $0.5 B S / \mathrm{km}^{2}$.

- The fading channel has an exponential distribution with the expected value of 1 .

- The user prefer a connection with two nearest BSs.

As shown in Fig. 2, the analytical result curves perfectly match with the points which represent the simulation results. This can verify the accuracy of the analytical results. Furthermore, the simulation results also indicates the density of BSs does not impose any impact on the network performance. This trend was discussed in Eq. 9. Take $K=2, \alpha=3$ for example, the average coverage probability is at when 0.7237 when $T=-4 \mathrm{~dB}$ and reduces by $24.1 \%$ to 0.5493 when $T=-2 \mathrm{~dB}$.

It is reminded that the coverage threshold $T$ represents the required SINR of the mobile user to successfully perform communication with its associated BS. Thus, when the coverage threshold increases, the average coverage probability significantly reduces as shown in Fig. 2.

It is observed from Fig. 2 that Joint Scheduling technique can significantly improve the average coverage probability of the typical user. Take $\alpha=3$ for example, when coverage threshold $T=2 \mathrm{~dB}$, the average coverage probability increases by $34.88 \%$ from 0.3908 to 0.5271 .

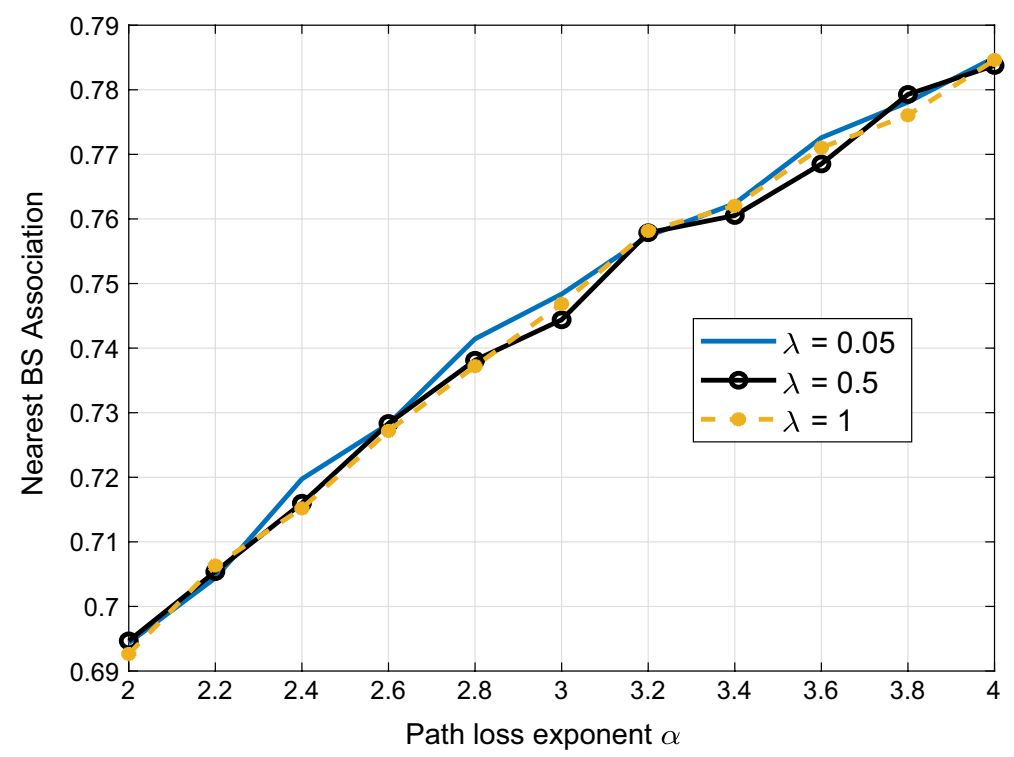

Fig. 3 Effects of path-loss exponent on the user association 


\subsection{User Association Analysis}

In this section, the effects of path loss exponent and the density of BSs on the user association is investigated. Figure 3 is obtained by Monte Carlo simulation. In this figure, the nearest BS association represents the probability in which the user connects to the nearest BSs.

It is observed from Fig. 3 that the user association probability is independent to the density of BSs. For example, when the density of BSs $\lambda$ increases from $\lambda=0.005$ to $\lambda=0.5$ then to $\lambda=1$ the nearest user association probability has a slight change between 0.7414 , 0.7381 and 0.7372 respectively. These numbers also show that the number of users associated with the second nearest BS are significant, up to around $25 \%$. This finding contradicts the conclusions in previous works [2] which stated that in the case of single-tier networks and the strongest association procedure is applied, all user will associate with the nearest BS when BSs transmit at the same power.

Figure 3 also indicates that the nearest user association increases with the path loss exponent. Take $\lambda=0.5$ for example, the user association probability increases by around $4 \%$ from 0.7283 to 0.7581 when the path loss exponent increases from 2.6 to 3.2. This phenomenon can be explained as follows: (1) The path loss of the signal is proportional to the distance from the user to its associated BS. (2) From the user perspective, the second nearest BS is farther than the nearest BS. Thus, the signal from the second nearest BS experiences a higher path loss than that from the nearest BS. Consequently, the user tends to associate with the nearest BS when the path loss exponent increases.

\section{Conclusion}

We investigated the average coverage probability of the typical user in the random cellular network using Joint Scheduling under Rayleigh fading condition. The general analytical results were conducted for Joint Scheduling network with the cluster of $K$. In the case of Joint Scheduling with the cluster size of $K=2$, the closed-form expression of user's average coverage probability is presented. The paper derived two important conclusions: (1) the probability in which the user connects to the nearest BS and the second nearest BS independents to the density of BSs. (2) The user connects to the nearest BS with a higher probability in the environment with high path loss exponent than that in the environment with low path loss exponent.

\section{References}

1. 3GPP TR 36.819 V11.1.0. (2011). Coordinated multi-point operation for LTE physical layer aspects.

2. Jo, H.-S., Sang, Y. J., Xia, P., \& Andrews, J. (2012). Heterogeneous cellular networks with flexible cell association: A comprehensive downlink SINR analysis. IEEE Transactions on Wireless Communications, 11(10), 3484-3495.

3. Singh, S., Dhillon, H. S., \& Andrews, J. G. (2013). Offloading in heterogeneous networks: Modeling, analysis, and design insights. IEEE Transactions on Wireless Communications, 12(5), 2484-2497.

4. Bassoy, S., Farooq, H., Imran, M. A., \& Imran, A. (2017). Coordinated multi-point clustering schemes: A survey. IEEE Communications Surveys Tutorials, 99, 1-1.

5. ElSawy, H., Hossain, E., \& Haenggi, M. (2013). Stochastic geometry for modeling, analysis, and design of multi-tier and cognitive cellular wireless networks: A survey. IEEE Communications Surveys and Tutorials, 15(3), 996-1019. 
6. Jung, S. Y., Lee, H. K., \& Kim, S. L. (2013). Worst-case user analysis in Poisson Voronoi cells. IEEE Communications Letters, 17(8), 1580-1583.

7. Moltchanov, D. (2012). Survey paper: Distance distributions in random networks. Ad Hoc Networks, 10(6), 1146-1166. https://doi.org/10.1016/j.adhoc.2012.02.005.

8. Andrews, J. G., Baccelli, F., \& Ganti, R. K. (2010). A new tractable model for cellular coverage. In 2010 48th Annual Allerton conference on communication, control, and computing (Allerton) (pp. 1204-1211).

9. Lam, S. C., Sandrasegaran, K., \& Ghosal, P. (2017). Performance analysis of frequency reuse for PPP networks in composite Rayleigh-lognormal fading channel. Wireless Personal Communications, 96, 989.

Publisher's Note Springer Nature remains neutral with regard to jurisdictional claims in published maps and institutional affiliations.

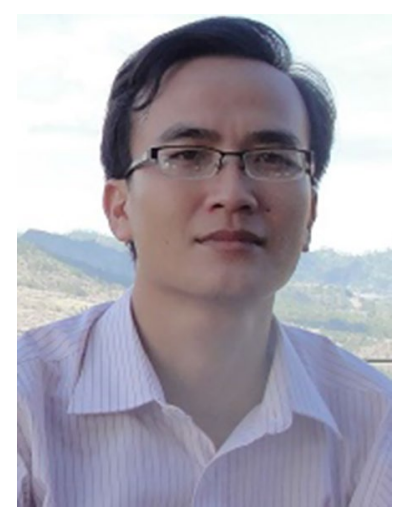

Dr. Sinh Cong Lam is a senior lecturer at VNU University of Engineering and Technology (VNU UET). He hold a Ph.D in Engineering from University of Technology Sydney Australia (2018), a Master in Electronics (2012) and a Bachelor in Electronics - Telecommunications (2010) from VNU UET. His research work focuses on Long-Term Evaluation (LTE) networks, especially Performance Analysis and Inter-Cell Interference Management techniques.

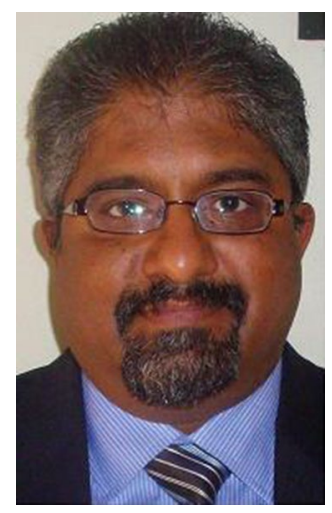

Dr. Kumbesan Sandrasegaran is an Associate Professor at UTS and Centre for Real-Time Information Networks (CRIN). He holds a Ph.D. in Electrical Engineering from McGill University (Canada) (1994), a Master of Science Degree in Telecommunication Engineering from Essex University (1988) and a Bachelor of Science (Honours) Degree in Electrical Engineering (First Class) (1985). His current research work focuses on two main areas (a) radio resource management in mobile networks, (b) engineering of remote monitoring systems for novel applications with industry through the use of embedded systems, sensors and communications systems. He has published over 100 refereed publications and 20 consultancy reports spanning telecommunication and computing systems. 